\title{
Rising to the challenge - Reports from APE 2018, held in Berlin ${ }^{1}$
}

\author{
Chris Armbruster* \\ Director Community, The Drivery - Mobility Innovators' Club Berlin, Germany
}

For one of Europe's most important gatherings on scholarly communication, 'Academic Publishing in Europe', more than 250 delegates gathered in Berlin from 16 to 17 January (pre-conference 15 January). Since 2006, the conference series has addressed key challenges, particularly technological innovation and business models.

'Publishing 2020: Ramping up relevance' was the focus this year, and the notion of ramping up relevance led to the re-affirmation of key themes that have characterised the conference series, and made it valuable to those present:

1. Open access; and particularly funder demands for more progress on the transition to open access publishing by national deals to enable the so-called 'flipping' of the business model to avoid 'doubledipping' by publishers when collecting subscription fees, plus article processing charges.

2. Content versus data as an unresolved issue for many publishers that remain undecided as to whether they should keep prioritising peer review and publication of content, or focus on providing a platform capable of creating value from data.

3. Looming new technological challenges associated with the blockchain and the rise of machine learning that is likely to challenge academic publishing.

Open access (OA) returned to the fore in an opening address delivered by the President of the Berlin Brandenburg Academy of Science and Humanities (also the conference venue), Professor Dr Martin Grötschel. He took the opportunity to convey his impressions of the standoff between the German libraries and research organisations and Elsevier, having taken part in the negotiations. The president of the International Publishers Association, Dr Michiel Kolman, could not respond, as he had delivered his opening address earlier.

Open access as public policy was a major theme at the pre-conference. Geraldine Clement-Stoneham (MRC, UK) detailed how the openness of research results has been a priority of public policy for more than a decade, and how funders expect more progress on open access publishing and open data. Robert van der Vooren (VSNU) outlined the ambition of 100 per cent open access to journal publications in 2020 by funding article-processing charges. To date, deals with the top eight publishers have led to more than 50 per cent open access publishing for about 24,000 corresponding authors funded by VSNU institutions. Axel Marion (Swiss Rectors' Conference), presented an open access strategy by which all publicly-funded

\footnotetext{
*E-mail: Chris.Armbruster@EUI.eu.

${ }^{1}$ Reprinted with kind permission of 'Research Information' (c) 2018.
} 
research results will be open access by 2024. A comprehensive action plan for SFr30m supports adoption of open access, reform of copyright, experiments with publication formats and so on. In parallel, the Swiss National Science Foundation has achieved about 50 per cent open access and is pushing for 100 per cent by 2020 .

In a keynote, Jean-Claude Burgelman (EC DG RTD) highlighted that in 2018 the building of a complementary OA publishing platform will be launched by tender. For H2020 grantees, it will provide fast publication by pre-prints, support open peer review and next-generation metrics. In parallel, a pilot is being launched for data, the European Open Science Cloud.

Particularly noteworthy on OA was a measured speech by David Sweeney (HEFCE, UK; executive chair designate 'Research England'). While the delivery was diplomatic, the message was clear. Whereas the UK path had been to lead through a partnership between funders and publishers, this partnership was not delivering a transition to OA publishing, and there are doubts all publishers are still on board as partners. Unless measurable success becomes evident soon, public funders would need to reconsider their approach to open access publishing.

There is a connection between open access and the debate around content versus data. Open access means 'releasing' the content, and there is a perception among publishers that they may lose control over the content and the data. This became particularly evident in the session 'All about piracy' among the presenters and discussants, Wouter Haak (Elsevier), Dr Duncan Campbell (Wiley), Wim van der Stelt (Springer Nature), and Charlie Rapple (Kudos). Sci-Hub is believed to have copied almost the entire corpus, driving publishers to re-assert the importance of content. Sharing platforms, like ResearchGate, have accumulated much data on users and publications. While Sci-Hub has been used, but is out of reach; various publisher consortia are negotiating with ResearchGate (e.g. Springer Nature) or, alternatively, issuing take-down notices (e.g. Elsevier, Wiley).

The APE Lecture by Dr Annette Thomas (Clarivate Analytics) outlined a comprehensive data-driven approach that puts the researcher at the centre. A 360 Framework is used to capture the impact of all kinds of publications, plus covering the workflow (e.g. resources, data) and addressing the services researchers provide (e.g. peer review, teaching).

The blockchain session focused on its potential for disintermediation of publishing services. The notion that the blockchain is decentralised, distributed, and immutable, leads protagonists to believe it could be the foundation of a more integrated open system, a kind of single, transparent repository supporting the whole scholarly cycle encompassing research, publication and communication. While applicability of blockchain was discussed, product prototypes with user traction have yet to emerge.

The session on AI and publishing shifted between the more general claim machine learning may be utilised to redesign the scholarly workflow, and the presentation of ideas for specific applications, e.g. matching papers and reviewers for peer review. While products with machine learning inside have been developed, the session made clear current uptake is limited. Both the blockchain and machine learning have not yet had much practical impact in scholarly publishing. 